Published in final edited form as:

J Am Chem Soc. 2019 July 24; 141(29): 11489-11496. doi:10.1021/jacs.9b02453.

\title{
The reactivity of an unusual amidase may explain colibactin's DNA cross-linking activity
}

\author{
Yindi Jiang ${ }^{\dagger}$, Alessia Stornetta ${ }^{\ddagger}$, Peter W. Villalta ${ }^{\ddagger}$, Matthew R. Wilson ${ }^{\dagger} \S$, Paul D. \\ Boudreau $^{\dagger}$, Li Zha ${ }^{\dagger, \|}$, Silvia Balbo ${ }^{\ddagger}$, Emily P. Balskus ${ }^{\star}, \dagger$ \\ tDepartment of Chemistry and Chemical Biology, Harvard University, 12 Oxford Street, \\ Cambridge, MA 02138, United States \\ ¥Masonic Cancer Center, University of Minnesota, 2231 Sixth Street Southeast, Minneapolis, MN \\ 55455, United States
}

\begin{abstract}
Certain commensal and pathogenic bacteria produce colibactin, a small molecule genotoxin that causes interstrand cross-links in host cell DNA. Though colibactin alkylates DNA, the molecular basis for cross-link formation is unclear. Here, we report that the colibactin biosynthetic enzyme $\mathrm{ClbL}$ is an amide bond-forming enzyme that links aminoketone and $\beta$-keto thioester substrates in vitro and in vivo. The substrate specificity of ClbL strongly supports a role for this enzyme in terminating the colibactin NRPS-PKS assembly line and incorporating two electrophilic cyclopropane warheads into the final natural product scaffold. This proposed transformation was supported by the detection of a colibactin-derived cross-linked DNA adduct. Overall, this work provides a biosynthetic explanation for colibactin's DNA cross-linking activity and paves the way for further study of its chemical structure and biological roles.
\end{abstract}

\section{Graphical Abstract}

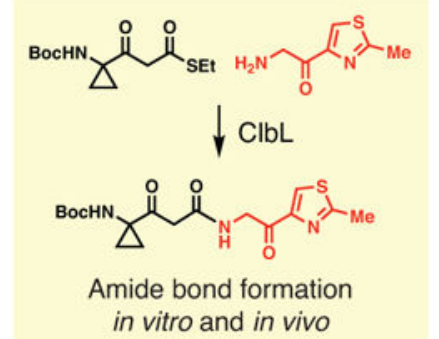

in vitro and in vivo

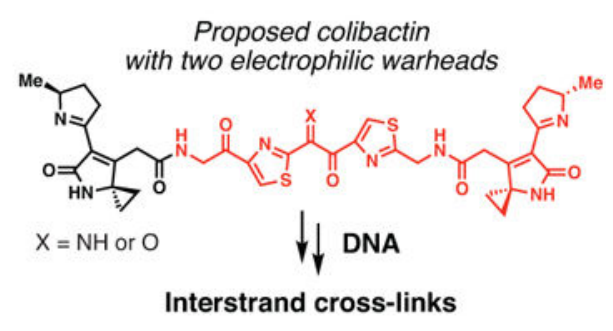

"Corresponding Author: balskus@chemistry.harvard.edu.

§Vertex Pharmaceuticals, 50 Northern Ave, Boston, MA 02210, United States.

"Boston Children's Hospital, 300 Longwood Avenue, Boston, MA 02115, United States.

Supporting Information

The Supporting Information is available free of charge on the ACS Publications website.

Materials and Methods for enzyme purification, synthetic procedures, and additional experimental data (PDF)

The authors declare no competing financial interest. 


\section{INTRODUCTION}

Colibactin is a genotoxin made by human gut commensal and extraintestinal pathogenic Escherichia coli strains and other Proteobacteria. ${ }^{1}$ Colibactin-producing E. coli $\left(p k s^{+} E\right.$. coli) cause DNA double strand breaks, ${ }^{1-2}$ affect progression of colitis-associated colorectal cancer (CRC) in mouse models, ${ }^{3-4}$ and are more frequently detected in patients with colorectal cancer. ${ }^{5-7}$ Recent studies have indicated colibactin's genotoxicity likely arises from a direct interaction with DNA, as we and others have reported the accumulation of interstrand cross-links in human cell lines incubated with $\mathrm{pks}^{+}$E. coli. ${ }^{8,9}$ Cell lines with impaired interstrand cross-link repair are also more sensitive to colibactin exposure. ${ }^{8}$ However, the underlying chemical and enzymatic basis for colibactin's DNA cross-linking activity remains unclear.

Colibactin is produced by a 54-kb gene cluster (the $p k s$ island) encoding a nonribosomal peptide synthetase-polyketide synthase (NRPS-PKS) hybrid assembly line, ${ }^{1}$ and its biosynthesis involves a self-protection mechanism in which an $N$-acyl-D-asparagine scaffold (prodrug motif) is initially assembled and elaborated to produce an inactive metabolite (precolibactin) (Figure 1A). ${ }^{10-12}$ Precolibactin is cleaved by the periplasmic peptidase ClbP to release the active colibactin genotoxin. This active species has not yet been identified, isolated, or structurally characterized, presumably due to its instability. We and others have isolated and structurally characterized several stable candidate precolibactins (1-5) from $p k s$ ${ }^{+}$E. coli strains lacking ClbP (Figure 1B). ${ }^{13-20}$ Notably, metabolites $\mathbf{2 - 5}$ contain a cyclopropane ring, a structural feature found in some DNA-alkylating agents. ${ }^{21-22}$ Formation of an $\alpha, \beta$-unsaturated imine after cleaving the prodrug motif in precolibactin likely enhances the electrophilicity of the cyclopropane toward DNA bases, and the recent identification and structural characterization of colibactin-derived DNA adducts in human cells and colonic epithelial cells provided support for this functional group serving as an electrophilic warhead. ${ }^{9,23}$ However, it was unclear how this structural motif could lead to generation of an interstrand cross-link.

To address this question, we focused on understanding previously uncharacterized components of the colibactin biosynthetic enzymes. Efforts to elucidate the biosynthetic origins of isolated candidate precolibactins have led to putative functional assignments for all of the enzymes in the biosynthetic pathway except for the putative amidase ClbL. This enzyme is essential for the genotoxicity of $\mathrm{pks}^{+} E$. coli ${ }^{1}$ but is not required for biosynthesis of any isolated candidate precolibactins reported to date, indicating that these metabolites were likely not precursors of the final genotoxin(s). A recent finding that $p k s^{+} E$. coli strains with inactive ClbL were incapable of cross-linking DNA suggested that this enzyme participates in producing the active cross-linking agent. ${ }^{24}$ However, the molecular basis for ClbL's contribution to colibactin's cross-linking activity is unknown.

Here, we elucidate the function of ClbL in colibactin biosynthesis. By identifying, isolating, structurally characterizing a ClbL-dependent candidate precolibactin from $\mathrm{pks}^{+} \mathrm{E}$. coli lacking $\mathrm{ClbP}$ and reconstituting its biosynthesis in vitro, we show that $\mathrm{ClbL}$ is an amide bond-forming enzyme. ClbL couples $\beta$-keto thioesters and aminoketones, structural motifs known to be generated by the colibactin assembly line. Based on this observation, we 
hypothesize that $\mathrm{ClbL}$ assembles a pseudo-dimeric precolibactin precursor possessing two electrophilic cyclopropane warheads which upon ClbP activation can cross-link DNA. This proposal is supported by the detection of two new colibactinderived DNA adducts in $p k s^{+} E$. coli-treated DNA samples, including a putative cross-link. Overall, this work reveals the activity of ClbL and provides a molecular explanation for colibactin's DNA cross-linking activity.

\section{Results}

ClbL belongs to the amidase signature (AS) family of enzymes, which contain a Ser-cisSerLys catalytic triad (Figure S1). ${ }^{25}$ These enzymes catalyze a wide variety of hydrolytic reactions, including the breakdown of the neurotransmitter anandamide by fatty acid amide hydrolase (FAAH) in humans and hydrolysis of glutamine by Glu-tRNA Gln amidotransferase subunit A (GatA) in bacteria. ${ }^{26-27}$ Phylogenetic analysis of representative amidases indicated that $\mathrm{ClbL}$ is part of a distinct clade, potentially implying functional differences (Figure S2). Alignment of ClbL with structurally characterized amidases revealed the three conserved, essential catalytic residues (K80, S155, and S179) (Figure S1). To test whether these active site amino acids are essential for ClbL function and genotoxicity, plasmids expressing versions of $\mathrm{ClbL}$ with these residues individually mutated to alanine were constructed and used to complement a strain of $p k s^{+} E$. coli lacking $c l b L$ (BACpks $\Delta c l b L$ ). These mutant strains failed to cause cell cycle arrest in HeLa cells (Figure S1), suggesting that these three residues are crucial for the function of ClbL and confirming that ClbL's catalytic activity is critical for genotoxicity.

To identify candidate precolibactins linked to ClbL, we overexpressed wild-type ClbL or the ClbL active site mutants in E. coli DH10B BACpks $\Delta c l b P / \Delta c l b L$. Methanol extracts from these cultured strains were compared to identify mass features affected by the activity of ClbL. As amidases are typically amide bond-hydrolyzing enzymes, we expected the substrate for ClbL to accumulate in the mutant strains. Surprisingly, we were unable to detect any up-regulated metabolites in these strains. Consistent with the previous findings, ${ }^{16}$ ClbL mutant strains produced detectable levels of $\mathbf{2}$ and other known candidate precolibactins (Figure S3), confirming that these metabolites are not ClbL-dependent. We did observe a novel metabolite $(\mathbf{6}, \mathrm{m} / \mathrm{z} 729.4334)$ accumulate only in strains expressing wild-type ClbL (Figure 2A, Table S4). To confirm metabolite 6 was pks-associated, we fed wild-type ClbL-expressing strains L- $\left[1-{ }^{13} \mathrm{C}\right]$ Met, the amino acid precursor of the cyclopropane ring. ${ }^{15,16}$ Observation of a +1 mass shift demonstrated that methionine is incorporated into 6 (Figure 2B). Moreover, MS/MS fragmentation analysis indicated that 6 contains the prodrug scaffold $(\mathrm{m} / \mathrm{z} 324.2437)^{18}$ and a colibactin-characteristic fragment ion $(\mathrm{m} / \mathrm{z} 231.1119)^{17,28}$ (Figure S4). These results suggested that metabolite 6 is a novel ClbLdependent candidate precolibactin.

We then isolated and structurally characterized this new metabolite, obtaining approximately $0.7 \mathrm{mg}$ of purified 6 from $140 \mathrm{~L}$ of an E. coli DH10B BACpks $\Delta c l b P$ strain overexpressing ClbL. The high-resolution mass of this compound $\left([\mathrm{M}+\mathrm{H}]^{+}=729.4334\right)$ yielded a molecular formula of $\mathrm{C}_{41} \mathrm{H}_{56} \mathrm{~N}_{6} \mathrm{O}_{6}$. One-dimensional (1D) and two-dimensional (2D) NMR along with 
MS/MS fragmentation analysis allowed us to elucidate its chemical structure (Figure 3A, Figure S5-S8, Table S5).

Similarities between the NMR spectra of known precolibactins and metabolite $\mathbf{6}$ enabled us to assign fragments I and II easily (Figure 3B). Unexpectedly, COSY and HMBC correlations indicated the presence of an indole ring (Figure 3B). We were unable to assign the precise locations of a methylene and carbonyl within 6 using NMR, but MS/MS fragmentation analysis revealed a loss of indole $(\mathrm{m} / \mathrm{z}$ 117.0607) from the parent ion, suggesting that the ketone is directly attached to the indole ring (Figure S9). To further support this structural assignment, we chemically synthesized a standard of metabolite $\mathbf{6}$. This standard possessed the same exact mass, MS/MS fragmentation pattern, retention time, ${ }^{1} \mathrm{H}$ spectrum, and UV spectrum as 6 isolated from bacterial strains (Figure 3C, Figure S10S12), confirming our structural assignment.

The chemical structure of $\mathbf{6}$ guided subsequent efforts to elucidate the function of ClbL. Metabolite $\mathbf{6}$ does not contain either a free amine or a carboxylic acid, which are the functional groups liberated by the activity of characterized amidases. Based on the known steps of colibactin biosynthesis, we envisioned two possible pathways for the formation of 6 (Figure 4A). In pathway A, the intermediate generated by ClbJ-NPRS 1 ${ }^{17,18}$ could be intercepted and offloaded via nucleophilic attack by the known E. coli metabolite indole. ${ }^{29}$ In pathway B, 2-amino-1-(1H-indol-3-yl)ethan-1-one (7) could act as a nucleophile to offload the $\beta$-keto thioester intermediate generated by the upstream biosynthetic enzyme ClbI. ${ }^{30}$

To test the involvement of pathway A, we made multiple double mutants in E. coli DH10B BACpks $\Delta c l b P$ (Figure 4B). Detection of $\mathbf{6}$ in the absence of $\mathrm{ClbJ}$ and $\mathrm{ClbP}$ indicated that $\mathrm{ClbJ}$ was not required for its biosynthesis. To confirm this result, we fed $\left[1,2-{ }^{13} \mathrm{C}_{2}\right] \mathrm{Gly}$, the building block used by ClbJ-NPRS1, to a glycine auxotrophic BAC $p k s \Delta c l b P$ strain. We observed a +2 mass shift in the known glycine-derived precolibactin 3, but not in 6 (Figure S13), confirming that pathway A is not the source of 6 .

We next explored the involvement of pathway B by reconstituting the biosynthesis of metabolite 6 in vitro. We cloned, overexpressed, and purified C-terminal His $_{6}$-tagged ClbL (Figure S14). An assay mixture containing Sfp, ClbN, ClbB, ClbC, ClbH, ClbI, ClbL, myristoyl-CoA, malonyl-CoA, L-Asn, L-Ala, NADPH, ATP, SAM, and 7 generated 6 (Figure 4C, trace iv). To further confirm the involvement of $\mathbf{7}$ in this process, $\mathbf{7}$ was fed to an E. coli DH10B BACpks $\Delta c l b P$ strain overexpressing ClbL. An approximately 20 -fold increase in the production of 6 was observed (ion count of $4 \times 10^{4}$ vs. $7 \times 10^{5}$, Figure $4 D$ ), consistent with pathway B being operative in vivo. Notably, these results suggested that ClbL catalyzes amide bond formation rather than amide bond hydrolysis.

To further characterize the activity of ClbL in vitro, we synthesized ethyl thioester $\mathbf{8}$ as a surrogate for the ClbI-tethered biosynthetic intermediate (Figure 1A and 5A). Incubating $\mathrm{ClbL}$ with $\mathbf{7}$ and $\mathbf{8}$ (Figure 5A) revealed a single new product peak accumulating during an HPLC time course experiment (Figure 5B). We confirmed this product was amide 9 by comparison to a synthetic standard (Figure S15). 
To evaluate ClbL's preference for potential thioester substrates, we designed and synthesized ethyl thioester mimics of assembly line-tethered colibactin biosynthetic intermediates (Figure S16). ClbL and $\mathbf{7}$ were incubated with these mimics individually. In addition to $\mathbf{8}$, we found that thioesters $\mathbf{1 0}$ (a mimic for the ClbC-bound intermediate) and $\mathbf{1 1}$ (a mimic for the ClbJ-bound intermediate) could be recognized by ClbL (Figure 5C, Figure S17). To assess ClbL's ability to discriminate among these substrates, we conducted a competition experiment. Mixing $\mathbf{8}$ and 11, which are predicted to have very similar hydrophobicities, in equal molar amounts with $\mathrm{ClbL}$ and $\mathbf{7}$ produced more of the product arising from 8 compared to the product arising from 11 (Figure S17). This suggests ClbL preferentially recognizes the ClbI-bound intermediate. Furthermore, reexamination of extracts from ClbLoverexpressing strains did not reveal products of amide bond formation between $\mathbf{7}$ and ClbC- or ClbJ-tethered thioester intermediates (Figure S17). Together, these data indicate that ClbL prefers the ClbI-bound thioester as an electrophile for amide bond formation.

We next investigated the relevance of indole-containing nucleophile 7 to colibactin biosynthesis. $\mathbf{7}$ has only previously been found in termitomycamide $\mathrm{B}$, which was isolated from the giant mushroom Termitomyces titanicus (Figure S18). ${ }^{31}$ Intriguingly, 7 contains an a-aminoketone, a structural motif that could originate from decarboxylation of the essential colibactin building block aminomalonyl-ACP (Figure 5D). To examine the involvement of aminomalonyl-ACP biosynthetic enzymes in the production of 7, we used an E. coli DH10B $\mathrm{BAC} p k s \Delta c l b P \Delta c l b G$ strain, in which the transfer of aminomalonate to assembly line enzymes is abolished. This strain still produced $\mathbf{6}$, the candidate precolibactin derived from 7, indicating this aminomalonyl-ACP is not required (Figure 4B). We further showed that 7 does not originate from any of the pks island enzymes as it was detected in E. coli DH10B (Figure S19). 7 was also absent from uninoculated growth medium, indicating it is produced in E. coli endogenously. Therefore, we questioned whether the incorporation of $\mathbf{7}$ was relevant for the biosynthesis of the active colibactin genotoxin. Supplementing 7 to $p k s^{+} E$. coli during infection of host cells did not boost genotoxicity (Figure S20), suggesting that 7 is likely not a relevant substrate for ClbL in colibactin biosynthesis. We therefore hypothesize that metabolite $\mathbf{6}$ arises from the promiscuous activity of $\mathrm{ClbL}$ and is not an onpathway intermediate.

Next, we considered the possibility that an alternative nucleophile may be generated by colibactin biosynthetic enzymes. Assays with various amine substrates showed that the aaminoketone of $\mathbf{7}$ is crucial for recognition by ClbL. No products were observed when tryptamine replaced $\mathbf{7}$ in the in vitro reconstitution of the biosynthesis of $\mathbf{6}$. In contrast, we identified a new product $12(\mathrm{~m} / \mathrm{z} 690.4225)$ in assay mixtures containing 2aminoacetophenone in place of 7 (Figure 5E). This result suggested the $\mathrm{a}$-aminoketone and not the indole ring is important for substrate recognition by ClbL.

To identify the true nucleophilic coupling partner for ClbL, we examined its reactivity toward mimics of potential a-aminoketone substrates derived from the colibactin assembly line. We previously identified four PKS assembly line enzymes (ClbC, ClbI, ClbK, and $\mathrm{ClbO}$ ) that accept aminomalonate in vitro. ${ }^{18}$ Isolation of candidate precolibactin 5 also showed that the PKS module of ClbK accepts this building block in vivo, generating an a- 
aminoketone that is further oxidized to an a-iminoketone. ${ }^{19}$ Once offloaded from PKS modules, the immediate products of extension with aminomalonyl-ACP should readily undergo decarboxylation to give rise to a-aminoketones resembling 7 (Figure 5D). To examine whether $\mathrm{ClbL}$ can use assembly line derived $\mathrm{a}$-aminoketones for amide bond formation, we synthesized mimics of these potential substrates (13-15) and incubated them with ClbL and preferred thioester 8 (Figure S21). While ClbL can use all three mimics, it preferentially uses $\mathbf{1 5}$, which mimics the a-aminoketone derived from $\mathrm{ClbO}$, the final module of the colibactin assembly line (Figure 5C and 5F, Figure S22). Together, these results suggest a potential role for $\mathrm{ClbL}$ in colibactin biosynthesis: forming an amide bond between a ClbI-bound thioester and the a-aminoketone derived from $\mathrm{ClbO}$ (Figure 6).

This proposed assembly line logic has important implications for colibactin's biological activity. Amide bond formation by ClbL could link the precursors of two electrophilic cyclopropane warheads together. Cleavage of the two prodrug motifs by ClbP would generate a species that would contain two electrophilic warheads and could be capable of generating interstrand cross-links (17) (Figure 6). This metabolite could be the active colibactin genotoxin. This scenario is consistent with previous observations that $\mathrm{pks}^{+} \mathrm{E}$. coli promote interstrand cross-link formation in linearized plasmid DNA and human cell lines, ${ }^{8,9}$ as well as a recent finding that a synthetic 'colibactin mimic' containing two cyclopropane rings cross-links DNA in vitro, while a monomer cannot. ${ }^{32}$ This proposal is also consistent with our current understanding of colibactin-mediated DNA damage. We previously identified and characterized a pair of diasteromeric colibactin-derived DNA adducts (18) that likely arise from degradation of a larger lesion. ${ }^{9}$ Depurination of the proposed colibactinDNA cross-link 19 would give adduct $\mathbf{2 0}$, which could undergo oxidative cleavage to give monoadducts 21 and $\mathbf{1 8}$ (Figure 7A). A similar cleavage has been shown to occur readily in model substrates and a synthetic colibactin mimic. ${ }^{33,34}$

To test this proposal, we used targeted selection ion monitoring (SIM), untargeted adductomics, ${ }^{9}$ and $\mathrm{MS}^{2}$ analyses to identify and support the structures of these proposed colibactin-derived DNA adducts in linearized plasmid DNA treated with $p k s^{+} E$. coli. We identified a large DNA adduct consistent with cross-link $20(\mathrm{~m} / z$ 537.1719, $z=2)$ (Figure 7B). We also used untargeted DNA adductomics ${ }^{9}$ to find a cross-link adduct lacking one of the adenine moieties $22(\mathrm{~m} / \mathrm{z}$ 938.2821, $z=1 ; \mathrm{m} / \mathrm{z}$ 469.6447, $z=2)$ (Figure S23). To confirm 20 and 22 are $p k s$-associated, we supplemented assay mixtures with either L-[1-13 C]Met or L- $\left[1-{ }^{13} \mathrm{C}\right]$ Cys. The observation of +2 mass shifts in $\mathbf{2 0}$ and $\mathbf{2 2}$ suggested that they derive from two methionines and two cysteines (Figure 7C, Figure S23-24), supporting our proposal that colibactin contains two electrophilic warheads and two thiazoles. We also detected the proposed decomposition product 21 ( $\mathrm{m} / \mathrm{z}$ 568.1721) (Figure 7D-E, Figure S2527). Further characterization of colibactin-derived adducts will be necessary to clarify the identity of the active cross-linking agent.

\section{Conclusions}

ClbL belongs to the AS enzyme family, which participates in numerous biological processes, including the biosynthesis of the natural product actinonin in Streptomyces sp. ATCC 14903. ${ }^{35}$ In addition to catalyzing amide bond hydrolysis, some amidases exhibit 
acyltransferase activity. For example, the amidase from Rhodococcus sp. R312 transfers the acyl group of an amide to hydroxylamine at a 33-fold faster rate than the hydrolysis of the same amide, an activity that could be explained by the stronger nucleophilicity of hydroxylamine toward the acyl-enzyme intermediate compared to water. ${ }^{36}$ Our discovery of ClbL's activity expands the known roles of amidases to include interfacing with enzymatic assembly lines. It is intriguing that $\mathrm{ClbL}$ preferentially recognizes a-aminoketones over the corresponding primary amines. Future crystallographic studies may shed further light on this specificity.

We uncovered the activity of ClbL through studying the biosynthesis of metabolite $\mathbf{6}$, an offpathway candidate precolibactin that incorporates an endogenously produced $a$ aminoketone (7). A competition assay with 7 and 13-15 revealed that 7 is a preferred substrate for ClbL (Figure S22), explaining the presence of $\mathbf{6}$ in E. coli strains expressing the entire pks assembly line. However, we cannot conclude whether ClbL is likely to preferentially recognize 7 in vivo because we cannot examine its reactivity toward complex assembly line derived $a$-aminoketones. These proposed substrates are not readily accessed using chemical synthesis or in vitro reconstitution because of the instability of the aaminoketone located between the two thiazole heterocycles. ${ }^{33,34}$ Consistent with its instability, we were unable to detect a ClbO-derived a-aminoketone in vivo. Attempts to generate this substrate through in vitro reconstitution were also unsuccessful.

Nonetheless, ClbL's in vitro activity supports the hypothesis that this enzyme links two intermediates generated by the colibactin NRPS-PKS assembly line, a ClbI-bound thioester and a ClbO-derived a-aminoketone (Figure 6). This transformation may play a critical role in generating a DNA cross-linking agent that could represent the active colibactin genotoxin. This proposal is supported by ClbL's essential role in genotoxicity and cross-link formation, as well as our detection of adduct $\mathbf{2 0}$ in linearized plasmid DNA treated with $p k s^{+} E$. coli. We cannot exclude the possibility that $\mathrm{ClbL}$ accepts additional substrates. For example, we were unable to test whether $\mathrm{ClbL}$ could use a mimic of the putative ClbO-bound aminomalonate thioester intermediate as an electrophile due to its intrinsic instability. However, precedented colibactin biosynthetic logic and its DNA interstrand cross-linking activity allow us to establish two minimal criteria for the structure of colibactin: 1) it contains two electrophilic cyclopropane rings and 2) its construction requires all of the essential colibactin biosynthetic enzymes. Application of these conditions greatly narrows the potential structures for the active colibactin genotoxin(s) (Figure S28). These criteria also help in assessing the biological relevance of other reported candidate precolibactins. For example, Qian and Zhang recently disclosed a candidate precolibactin they proposed is the precursor to colibactin (Figure S29) ${ }^{20}$ Though the corresponding ClbP cleavage product appeared to damage DNA in vitro and in cells in a $\mathrm{Cu}^{2+}$-dependent manner, it did not form cross-links. It is also unclear whether $\mathrm{ClbL}$ is required for its biosynthesis. Together these observations suggest this metabolite may not be a major contributor to genotoxicity.

While this work was in revision, the Herzon and Crawford groups independently identified colibactin-derived cross-link adduct $\mathbf{2 0}$ providing further support for our model. ${ }^{34}$ They also chemically synthesized diketone $\mathbf{1 7}$ and showed that it cross-links linearized plasmid DNA at a concentration of $10 \mu \mathrm{M}$. This relatively low cross-linking reactivity suggests the active 
genotoxin(s) may contain the less stable but more reactive a-iminoketone motif. As our results suggest ClbL may be able to construct additional potential genotoxins (Figure S28), we feel that the final structure(s) of colibactin still requires further investigation.

In summary, these studies provide a biosynthetic rationale for the formation of interstrand DNA cross-links by the gut bacterial genotoxin colibactin. As such cross-links represent the most toxic form of DNA damage in human cells, it is possible this activity could explain the effects of $p k s^{+} E$. coli on tumor development. Future efforts to characterize additional precolibactins, colibactins, and colibactin-DNA adducts will provide us with the additional molecular information needed to decipher how colibactin influences CRC development in patients.

\title{
Supplementary Material
}

Refer to Web version on PubMed Central for supplementary material.

\section{ACKNOWLEDGMENT}

\begin{abstract}
We thank Alex Sieg for help with cloning, Lihan Zhang for help with NMR experiments, and Matthew Volpe for comments on the manuscript. We acknowledge financial support from National Institutes of Health (R01CA208834-02) (E. P. B). Salary support for P.W.V. was provided by the U.S. National Institutes of Health and National Cancer Institute [Grant R50-CA211256]. Mass spectrometry for DNA adduct analysis was carried out in the Analytical Biochemistry Shared Resource of the Masonic Cancer Center, supported in part by the U.S. National Institutes of Health and National Cancer Institute [Cancer Center Support Grant CA-77598]. M.R.W. acknowledges support from the American Cancer Society-New England Division Postdoctoral Fellowship PF-16-122-01-CDD.
\end{abstract}

\section{REFERENCES}

(1). Nougayrède J-P; Homburg S; Taieb F; Boury, Brzuszkiewicz E; Gottschalk G; Buchrieser C; Hacker J; Dobrindt U; Oswald E Escherichia coli induces DNA double-strand breaks in eukaryotic cells. Science 2006, 313, 848-851. [PubMed: 16902142]

(2). Cuevas-Ramos G; Petit CR; Marcq I; Boury M; Oswald E; Nougayrède J-P Escherichia coli induces DNA damage in vivo and triggers genomic instability in mammalian cells. Proc. Natl. Acad. Sci. U.S.A. 2010, 107, 11537-11542. [PubMed: 20534522]

(3). Arthur JC; Perez-Chanona E; Mühlbauer M; Tomkovich S; Uronis JM; Fan T-J; Campbell BJ; Abujamel T; Dogan B; Rogers AB; Rhodes JM; Stintzi A; Simpson KW; Hansen JJ; Keku TO; Fodor AA; Jobin C Intestinal inflammation targets cancer-inducing activity of the microbiota. Science 2012, 338, 120-123. [PubMed: 22903521]

(4). Arthur JC; Gharaibeh RZ; Mühlbauer M; Perez-Chanona E; Uronis JM; McCafferty J; Fodor AA; Jobin C Microbial genomic analysis reveals the essential role of inflammation in bacteriainduced colorectal cancer. Nat. Commun 2014, 5, 4724. [PubMed: 25182170]

(5). Buc E; Dubois D; Sauvanet P; Raisch J; Delmas J; Darfeuille-Michaud A; Pezet D; Bonnet R High prevalence of mucosa-associated E. coli producing cyclomodulin and genotoxin in colon cancer. PLoS ONE 2013, 8, e56964. [PubMed: 23457644]

(6). Eklof V; Löfgren-Burström A; Zingmark C; Edin S; Larsson P; Karling P; Alexeyev O; Rutegård J; Wikberg ML; Palmqvist R Cancer-associated fecal microbial markers in colorectal cancer detection. Int. J. Cancer 2017, 141, 2528-2536. [PubMed: 28833079]

(7). Dejea CM; Fathi P; Craig JM; Boleij A; Taddese R; Geis AL; Wu X; DeStefano Shields CE; Hechenbleikner EM; Huso DL; Anders RA; Giardiello FM; Wick EC; Wang H; Wu S; Pardoll DM; Housseau F; Sears CL Patients with familial adenomatous polyposis harbor colonic biofilms containing tumorigenic bacteria. Science 2018, 359, 592-597. [PubMed: 29420293] 
(8). Bossuet-Greif N; Vignard J; Taieb F; Mirey G; Dubois D; Petit C; Oswald E; Nougayrède J-P The colibactin genotoxin generates DNA interstrand cross-links in infected cells. mBio 2018, 9, e02393-17. [PubMed: 29559578]

(9). Wilson MR; Jiang Y; Villalta PW; Stornetta A; Boudreau PD; Carrá A; Brennan CA; Chun E; Ngo L; Samson LD; Engelward BP; Garrett WS; Balbo S; Balskus EP The human gut bacterial genotoxin colibactin alkylates DNA. Science 2019, 363, eaar7785. [PubMed: 30765538]

(10). Dubois D; Baron O; Cougnoux A; Delmas J; Pradel N; Boury M; Bouchon B; Bringer MA; Nougayrède J-P; Oswald E; Bonnet R ClbP is a prototype of a peptidase subgroup involved in biosynthesis of nonribosomal peptides. J. Biol. Chem 2011, 286, 35562-35570. [PubMed: 21795676]

(11). Brotherton CA; Balskus EP A prodrug resistance mechanism is involved in colibactin biosynthesis and cytotoxicity. J. Am. Chem. Soc 2013, 135, 3359-3362. [PubMed: 23406518]

(12). Bian X; Fu J; Plaza A; Herrmann J; Pistorius D; Stewart AF; Zhang Y; Müller R In vivo evidence for a prodrug activation mechanism during colibactin maturation. ChemBioChem 2013, 14, 1194-1197. [PubMed: 23744512]

(13). Vizcaino MI; Engel P; Trautman E; Crawford JM Comparative metabolomics and structural characterizations illuminate colibactin pathway-dependent small molecules. J. Am. Chem. Soc 2014, 136, 9244-9247. [PubMed: 24932672]

(14). Brotherton CA; Wilson M; Byrd G; Balskus EP Isolation of a metabolite from the pks island provides insights into colibactin biosynthesis and activity. Org. Lett 2015, 17, 1545-1548. [PubMed: 25753745]

(15). Vizcaino MI; Crawford JM The colibactin warhead cross-links DNA. Nat. Chem 2015, 7, 411417. [PubMed: 25901819]

(16). Bian XY; Plaza A; Zhang YM; Müller R Two more pieces of the colibactin genotoxin puzzle from Escherichia coli show incorporation of an unusual 1-aminocyclopropanecarboxylic acid moiety. Chem. Sci 2015, 6, 3154-3160. [PubMed: 28706687]

(17). Li ZR; Li Y; Lai JY; Tang J; Wang B; Lu L; Zhu G; Wu X; Xu Y; Qian PY Critical intermediates reveal new biosynthetic events in the enigmatic colibactin pathway. ChemBioChem 2015, 16, 1715-1719. [PubMed: 26052818]

(18). Zha L; Wilson MR; Brotherton CA; Balskus EP Characterization of polyketide synthase machinery from the pks island facilitates isolation of a candidate precolibactin. ACS Chem. Biol 2016, 11, 1287-1295. [PubMed: 26890481]

(19). Li ZR; Li J; Gu JP; Lai JYH; Duggan BM; Zhang WP; Li ZL; Li YX; Tong RB; Xu Y; Lin DH; Moore BS; Qian PY Divergent biosynthesis yields a cytotoxic aminomalonate-containing precolibactin. Nat. Chem. Biol 2016, 12, 773-775. [PubMed: 27547923]

(20). Li ZR; Li J; Cai W; Lai J; McKinnie S; Zhang WP; Moore B; Zhang W; Qian PY Macrocyclic colibactin induces DNA double-strand breaks via copper-mediated oxidative cleavage. bioRxiv 2019, doi: 10.1101/530204.

(21). Tanasova M; Sturla SJ Chemistry and biology of acylfulvenes: sesquiterpene-derived antitumor agents. Chem. Rev 2012, 112, 3578-3610. [PubMed: 22482429]

(22). Ghosh N; Sheldrake HM; Searcey M; Pors K Chemical and biological explorations of the family of CC-1065 and the duocarmycin natural products. Curr. Top. Med. Chem 2009, 9, 1494-1524. [PubMed: 19903166]

(23). Xue M; Shine E; Wang W; Crawford JM; Herzon SB Characterization of natural colibactinnucleobase adducts by tandem mass spectrometry and isotopic labeling. Support for DNA alkylation by cyclopropane ring opening. Biochemistry 2018, 57, 6391-6394. [PubMed: 30365310]

(24). Shine EE; Xue M; Patel JR; Healy AR; Surovtseva YV; Herzon SB; Crawford JM Model colibactins exhibit human cell genotoxicity in the absence of host bacteria. ACS Chem. Biol 2018, 13, 3286-3293. [PubMed: 30403848]

(25). Shin S; Lee TH; Ha NC; Koo HM; Kim SY; Lee HS; Kim YS; Oh BH Structure of malonamidase E2 reveals a novel Ser-cisSer-Lys catalytic triad in a new serine hydrolase fold that is prevalent in nature. EMBO J 2002, 21, 2509-2516. [PubMed: 12032064] 
(26). Cravatt BF; Giang DK; Mayfield SP; Boger DL; Lerner RA; Gilula NB Molecular characterization of an enzyme that degrades neuromodulatory fatty-acid amides. Nature 1996, 384, 83-87. [PubMed: 8900284]

(27). Curnow AW; Hong KW; Yuan R; Kim SI; Martins O; Winkler W; Henkin TM; Söll D GlutRNA $^{\text {Gln }}$ amidotransferase: a novel heterotrimeric enzyme required for correct decoding of glutamine codons during translation. Proc. Natl. Acad. Sci. U.S.A 1997, 94, 11819-11826. [PubMed: 9342321]

(28). Trautman EP; Healy AR; Shine EE; Herzon SB; Crawford JM Domain-targeted metabolomics delineates the heterocycle assembly steps of colibactin biosynthesis. J. Am. Chem. Soc 2017, 139, 4195-4201. [PubMed: 28240912]

(29). Li G; Young KD Indole production by the tryptophanase TnaA in Escherichia coli is determined by the amount of exogenous tryptophan. Microbiology 2013, 159, 402-410. [PubMed: 23397453]

(30). Zha L; Jiang Y; Henke MT; Wilson MR; Wang JX; Kelleher NL; Balskus EP Colibactin assembly line enzymes use S-adenosylmethionine to build a cyclopropane ring. Nat. Chem. Biol 2017, 13, 1063-1065. [PubMed: 28805802]

(31). Choi JH; Maeda K; Nagai K; Harada E; Kawade M; Hirai H; Kawagishi H Termitomycamides A to $\mathrm{E}$, fatty acid amides isolated from the mushroom Termitomyces titanicus, suppress endoplasmic reticulum stress. Org. Lett 2010, 12, 5012-5015. [PubMed: 20936815]

(32). Healy AR; Nikolayevskiy H; Patel JR; Crawford JM; Herzon SB A mechanistic model for colibactin-induced genotoxicity. J. Am. Chem. Soc 2016, 138, 15563-15570. [PubMed: 27934011]

(33). Healy AR; Wernke KM; Kim CS; Lees NR; Crawford JM; Herzon SB Synthesis and reactivity of precolibactin 886. ChemRxiv 2019, doi: 10.26434/chemrxiv.7849151.v1.

(34). Xue M; Kim CS; Healy AR; Wernke KM; Wang Z; Frischling MC; Shine EE; Wang W; Herzon SB; Crawford JM Structure elucidation of colibactin. bioRxiv 2019, doi: 10.1101/574053.

(35). Wolf F; Leipoldt F; Kulik A; Wibberg D; Kalinowski J; Kaysser L Characterization of the actinonin biosynthetic gene cluster. ChemBioChem 2018, 19, 1189-1195.

(36). Thiéry A; Maestracci M; Arnaud A; Galzy P Acyltransferase activity of the wide spectrum amidase of Brevibacterium sp. R312. J. Gen. Microbiol 1986, 132, 2205-2208. 


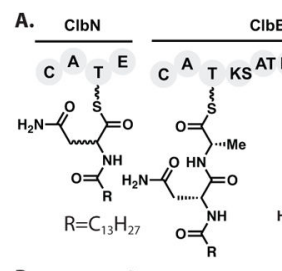

B.
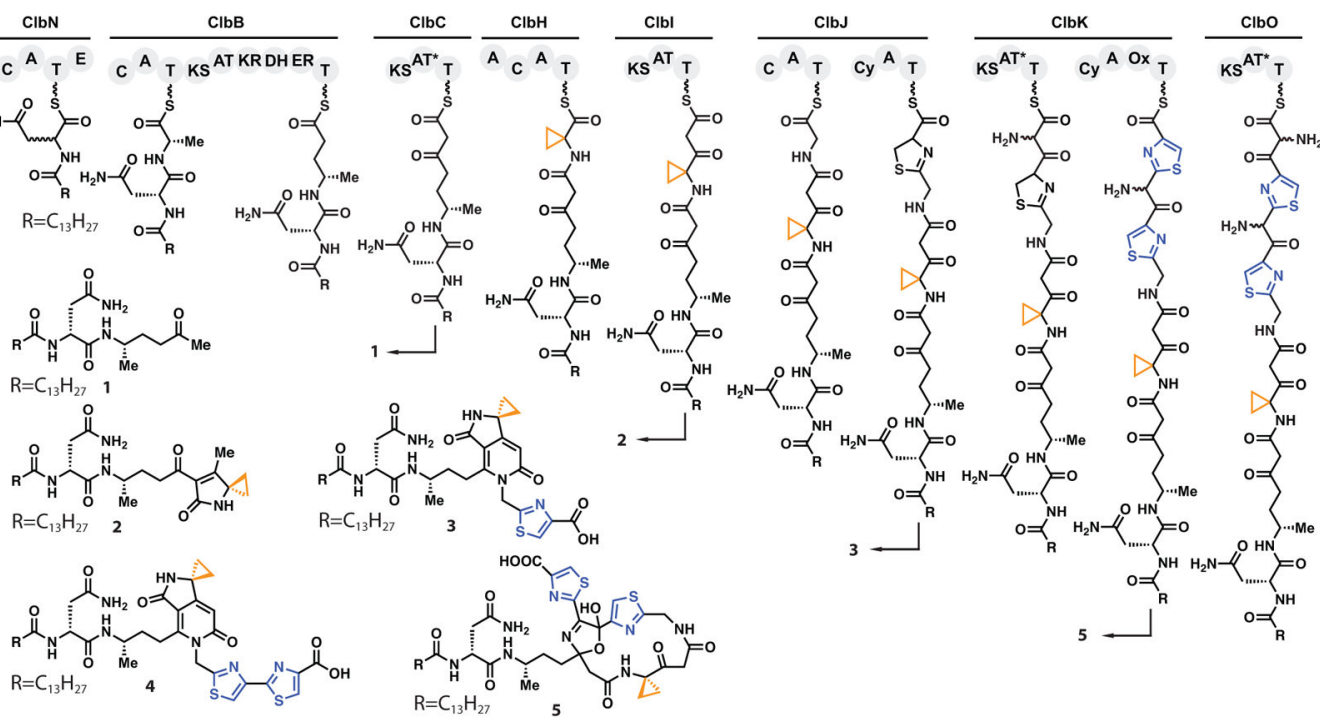

$\frac{\mathrm{ClbO}}{\mathrm{KS}^{\mathrm{AT}{ }^{*}}{ }_{\mathrm{T}}}$

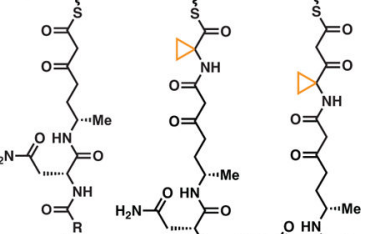

$0=$

${ }^{\mathrm{HN}}=0$
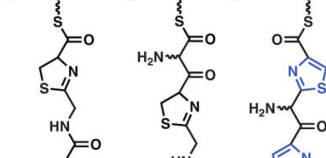

$\mathrm{O}=\int_{\mathrm{NHH}}^{\xi}$

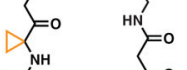
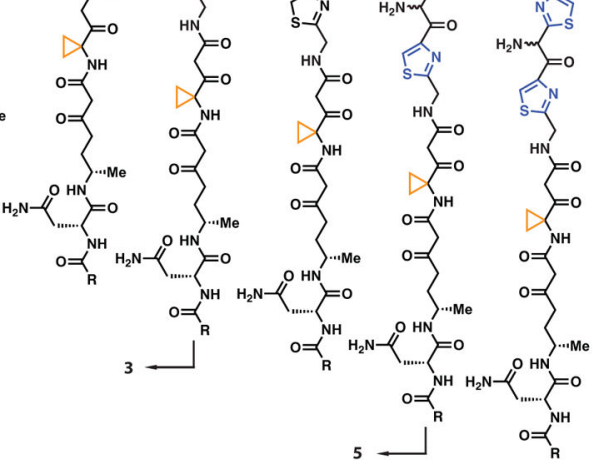

Figure 1. The pks enzymatic assembly line and the biosynthesis of characterized candidate precolibactins.

(A) Hypothetical biosynthetic pathway for candidate precolibactins 1-3 and 5. C, condensation; A, adenylation; T, thiolation; E, epimerization; KS, ketosynthase; AT, acyltransferase; KR, ketoreductase; DH, dehydratase; ER, enoyl reductase; $\mathrm{AT}^{*}$, atypical acyltransferase; $\mathrm{Cy}$, cyclization; Ox, oxidase. Cyclopropane rings are highlighted in orange. Thiazoles are highlighted in blue. (B) Chemical structures of isolated and characterized candidate precolibactins 1-5. 


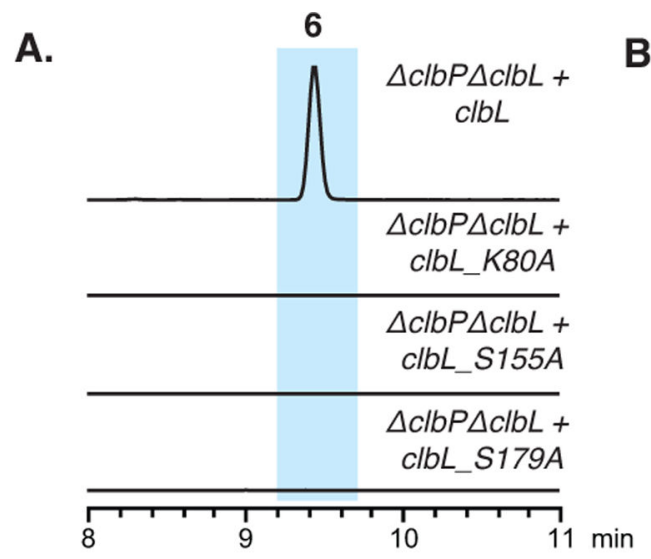

B. $\times 104$

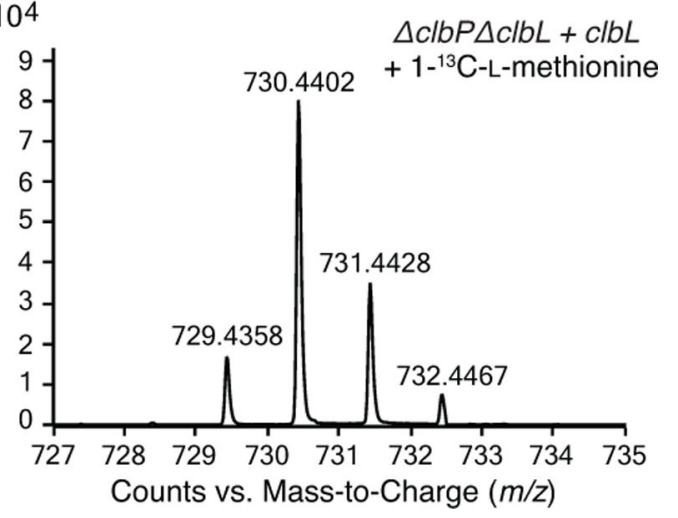

Figure 2. Discovery of a ClbL-dependent candidate precolibactin.

(A) Extracted ion chromatograms (EICs) of metabolite $6(\mathrm{~m} / \mathrm{z} 729.4334)$ in the extracts of $E$. coli expressing wild-type or mutant ClbL. (B) LC-MS analysis of feeding of $1{ }^{13} \mathrm{C}-\mathrm{L}-$ methionine to ClbL expressing E. coli strains. 
A.
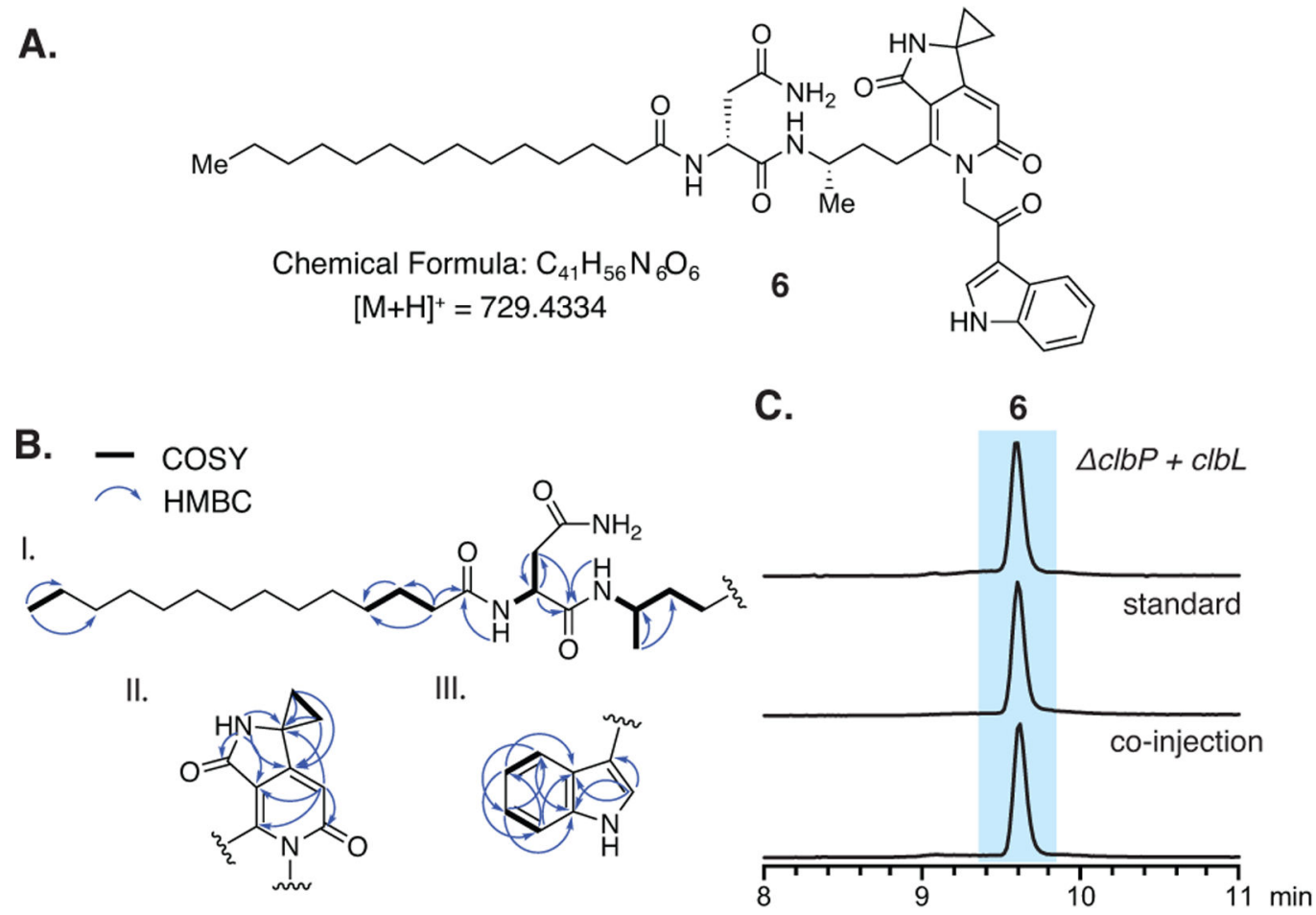

Figure 3. Structure determination of candidate precolibactin 6.

(A) Chemical structure of metabolite 6. (B) Key COSY and HMBC correlations that support this assignment. (C) EICs of metabolite $\mathbf{6}(\mathrm{m} / \mathrm{z}$ 729.4334) from the extracts of bacterial cultures, synthetic standard, and co-injection of synthetic standard and bacterial culture extracts. 


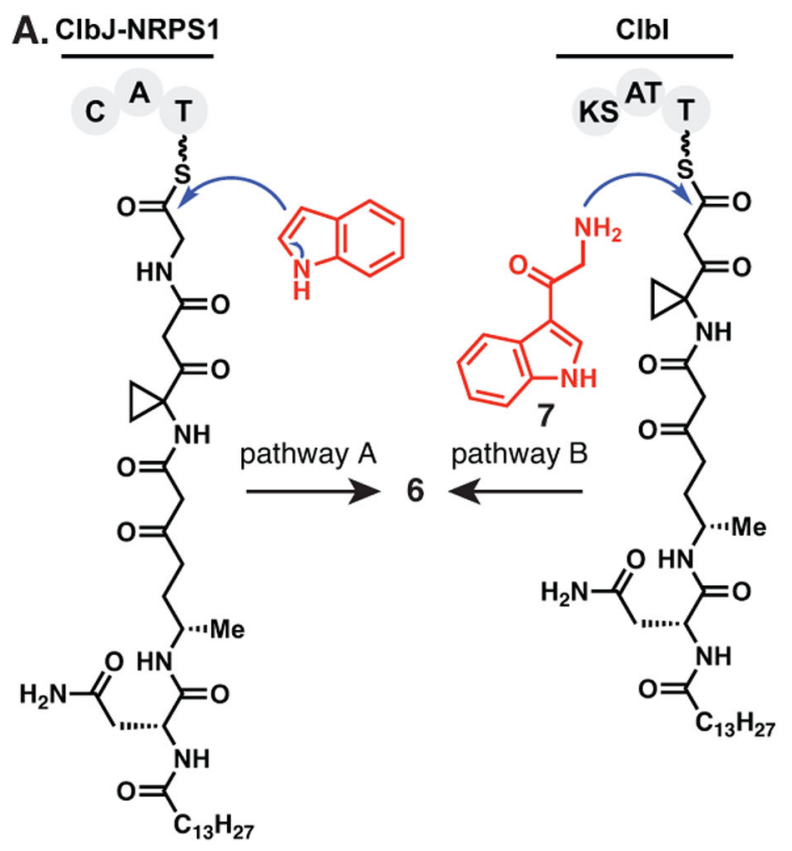

B. $\quad \begin{array}{ll}6 \\ 1\end{array} \quad \Delta c / b P$

C.

6

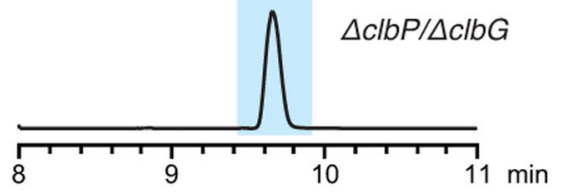

i: $\mathrm{Sfp}+\mathrm{ClbNBCHI}$

D.
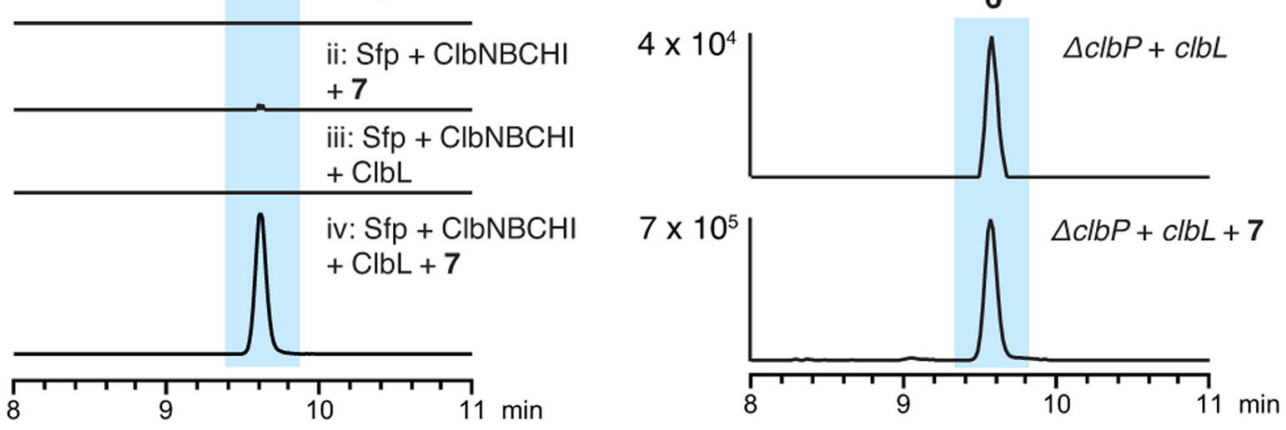

Figure 4. Investigating the formation of candidate precolibactin 6.

(A) Two proposed pathways could generate 6. (B) LC-MS analysis of extracts from double mutants of E. coli DH10B BACpks. (C) LC-MS analysis of in vitro reconstitution assays. (D) LC-MS analysis of cell pellet extracts from E. coli DH10B BACpks $\Delta c l b P+$ pTrc-clbL supplemented with 7. Panels B-D show the EICs of $6(\mathrm{~m} / \mathrm{z} 729.4334)$. 
A.

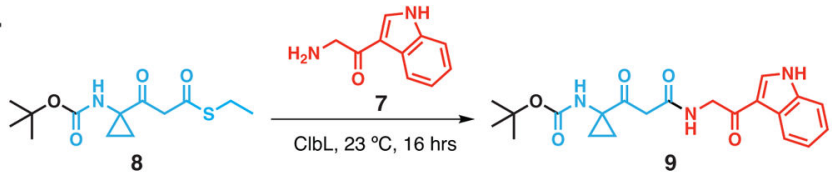

C. mimics of thioester-containing biosynthetic intermediates that are accepted by $\mathrm{ClbL}$

ClbC:

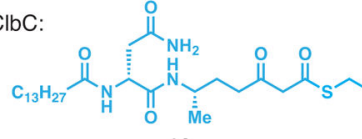
10

$\mathrm{Clbl}$

bl:<smiles>CCSC(=O)CC(=O)C1(NC(=O)OC(C)(C)C)CC1</smiles>

ClbJ:

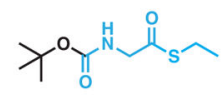

D.

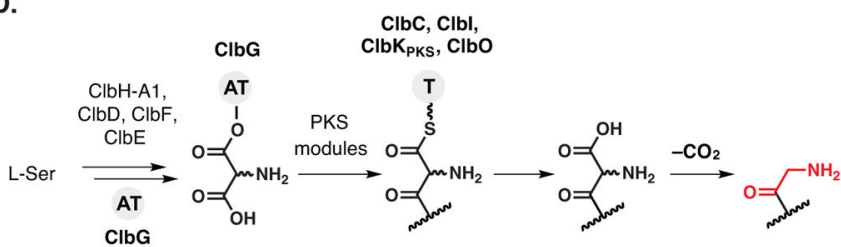

Clbl:

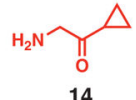

$\mathrm{ClbO}$

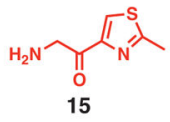

B.

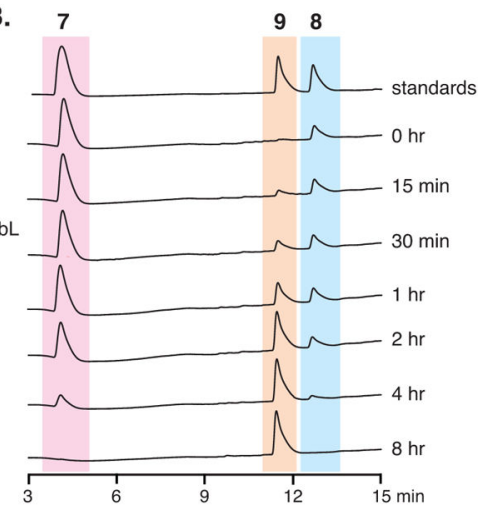

E.
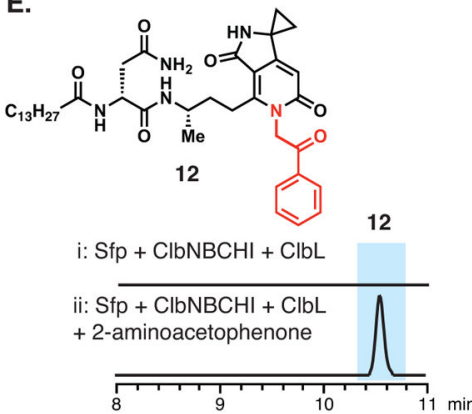

F.

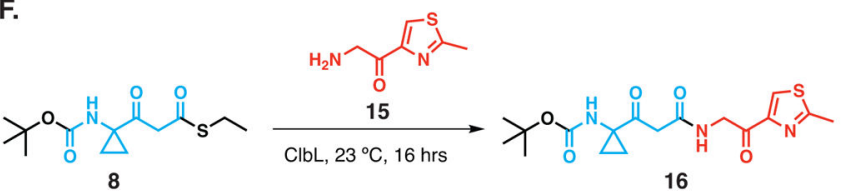

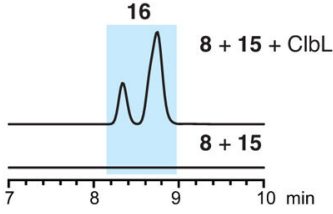

Figure 5. In vitro characterization of ClbL reveals its role in amide formation.

(A) Reaction scheme of an in vitro assay to form 9. (B) HPLC time course for the formation of 9 by ClbL (monitored at $250 \mathrm{~nm}$ ). (C) Thioesters and a-aminoketones accepted by ClbL. (D) The $p k s$ assembly line may produce a-aminoketone nucleophiles. (E) EICs of $\mathbf{1 2}(\mathrm{m} / \mathrm{z}$ 690.4225) formed in an in vitro reconstitution assay. (F) ClbL catalyzes the formation of $\mathbf{1 6}$ in vitro. The two peaks likely correspond to two tautomers of the b-ketoamide product. 


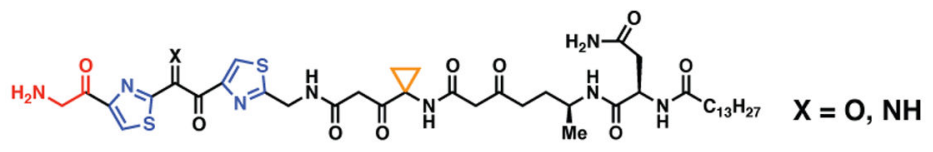

Decarboxylated elongation product of $\mathrm{ClbO}$

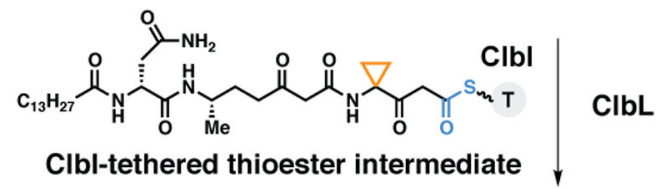

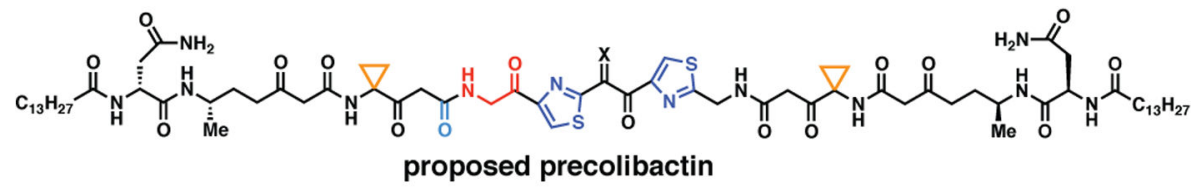
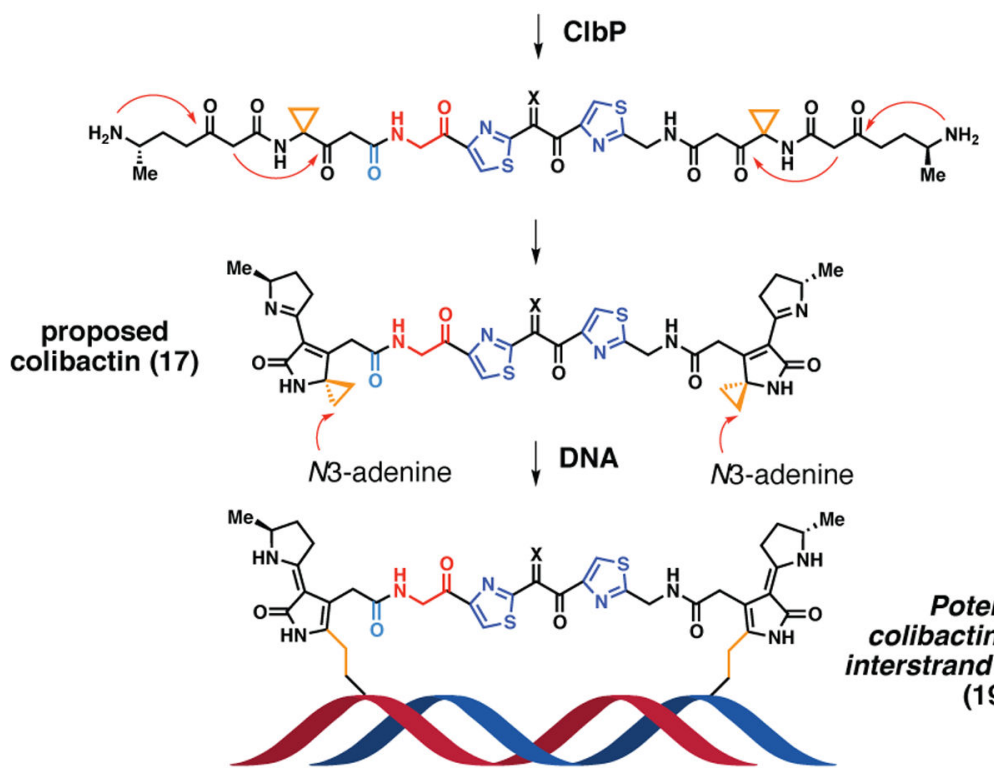

Potential colibactin-derived colibactin (17) interstrand cross-link

(19)

Figure 6. A model for ClbL-catalyzed formation of the active colibactin genotoxin). Key structural features colored as in Figures 1, 4, and 5. 
A.

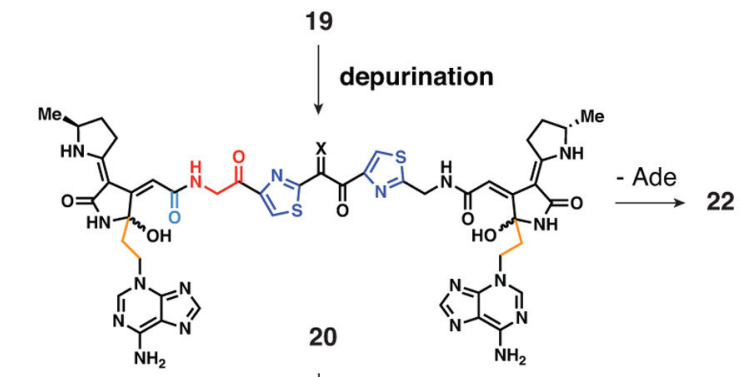

$\downarrow$
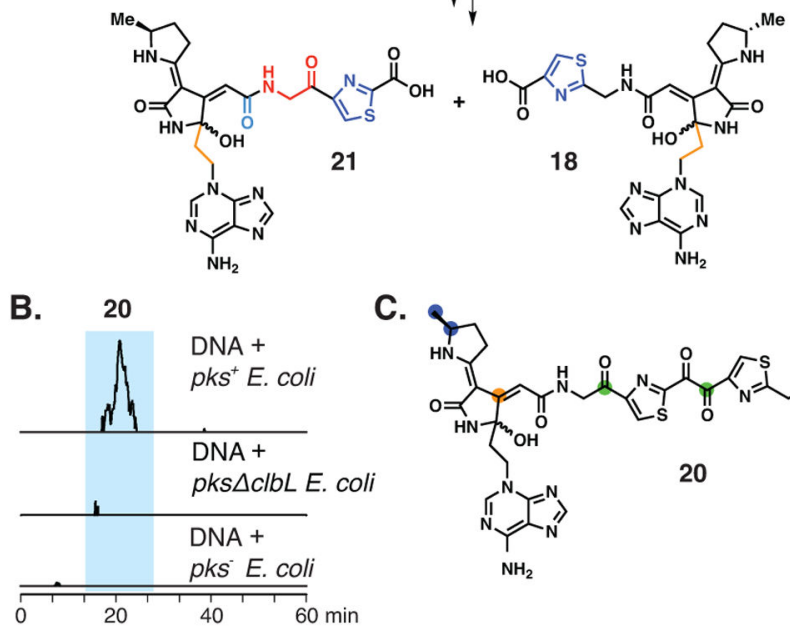

C.
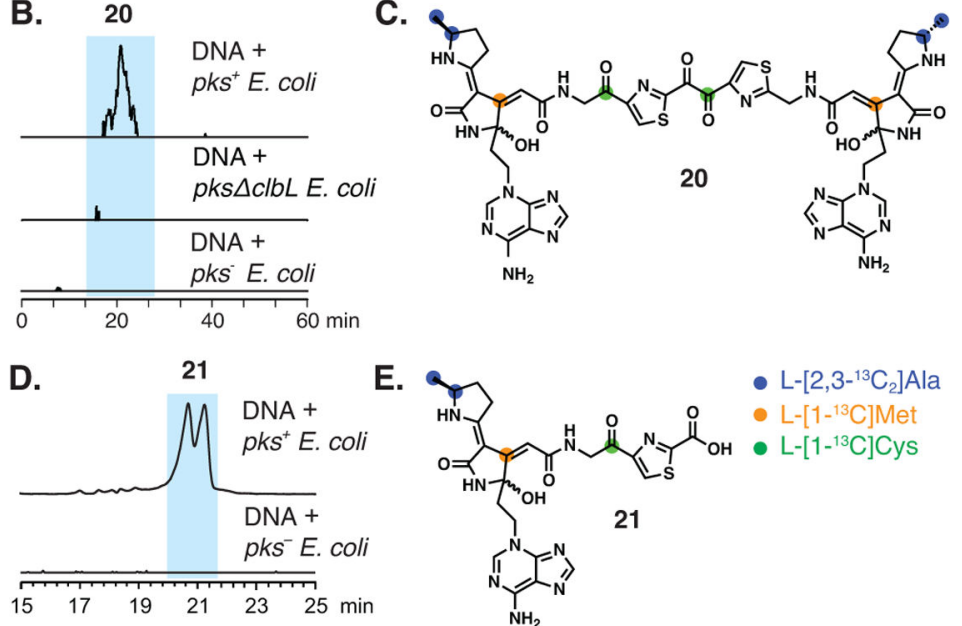

E.

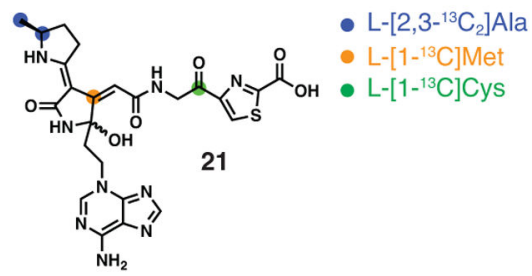

Figure 7. Detection of two new colibactin-derived DNA adducts.

(A) Predicted structures of colibactin-derived DNA adduct $\mathbf{2 0}$ and its decomposition products 21 and 18. (B) EICs of $\mathbf{2 0}(\mathrm{m} / z$ 537.1719, $z=2)$ in $p k s^{+} E$. coli treated DNA samples. (C) Adduct 20 incorporates two alanines, two methionines, and two cysteines. (D) EICs of $21\left(\mathrm{~m} / \mathrm{z}\right.$ 568.1721) in DNA samples treated with $\mathrm{pks}^{+} E$. coli. The two peaks likely arise from multiple diastereomers. (E) Adduct 21 incorporates one alanine, one methionine, and one cysteine. 